\title{
Off-Resonant Vibration Amplifier With Flattened Band-Pass Characteristic and Improved Axis Selectivity
}

\author{
Journal Article \\ Author(s): \\ Maiwald, Verena; Müller, Michelle; Ritz, Christian; Hierold, Christofer; Roman, Cosmin \\ Publication date: \\ 2017 \\ Permanent link: \\ https://doi.org/10.3929/ethz-b-000197692
}

Rights / license:

Creative Commons Attribution 3.0 Unported

Originally published in:

Journal of Microelectromechanical Systems 26(6), https://doi.org/10.1109/JMEMS.2017.2745051 


\title{
Off-Resonant Vibration Amplifier With Flattened Band-Pass Characteristic and Improved Axis Selectivity
}

\author{
Verena Maiwald, Michelle Müller, Christian Ritz, Christofer Hierold, Fellow, IEEE, and Cosmin Roman
}

\begin{abstract}
We present the design, fabrication, and characterization of an in-plane vibration sensor with frequency selective displacement amplification and differential capacitive read-out. The mechanical structure is based on six resonators with decreasing stiffness coupled in-plane. A differential capacitance attached to the last mass serves as electrical read out. Finite element and lumped element models are both presented. The devices were fabricated in a single mask silicon on insulatorbased process. The mechanical, as well as the capacitive transfer function and the pressure dependence, have been investigated experimentally and compared with simulations. The measured mean (minimum) amplification was $24 \mathrm{~dB}(16 \mathrm{~dB})$ over a bandwidth of $10 \mathrm{kHz}(3-13 \mathrm{kHz})$. While the mean amplification is pressure dependent, the minimum amplification and bandwidth show a less than $10 \%$ decrease over a wide pressure range from 6.3 to 64 mbar. The pressure dependent measurements also show that the minimum amplification is independent of the $Q$ factor of the modes down to values of $Q \sim 10$. Both simulation and experiment show that the off-axis modes occur outside the bandwidth of the device. Along with the low cross-sensitivity of the capacitive readout $(0.06 \%)$, this provides good axis selectivity despite the high number of degrees of freedom. The device can be used for detection of broadband vibration signals, e.g., for structural monitoring of infrastructure such as bridges and pipelines. [2017-0096]
\end{abstract}

Index Terms-Mechanical amplification, vibration sensor, coupled masses, axis selectivity, band-pass, multi-degree of freedom, mechanical filter, damping, mass-spring network.

\section{INTRODUCTION}

A COUSTIC emission (AE) and micro-seismic (MS) Structural Health Monitoring (SHM) in heterogeneous media requires the detection of broadband, weak signals. The vibrations emitted by structural damage (cracks) are short bursts of strain that propagate through the solid. Traditionally, the signals of interest lie in the range of tens up to several hundred $\mathrm{kHz}$ depending on the material and application. Recently, there has been increased interest in the detection of lower frequency $\mathrm{AE}$ signals from a few $\mathrm{kHz}$ upward with the purpose of increasing the measurement radius in high attenuation materials such as granular media, rock or concrete or in

Manuscript received April 24, 2017; revised July 12, 2017; accepted August 15, 2017. Date of publication September 11, 2017; date of current version November 29, 2017. This work was supported by the Swiss National Science Foundation through the Swiss Confederation under Nano-Tera.ch. Subject Editor Roger T. Howe. (Corresponding author: Verena Maiwald.)

The authors are with the Department of Mechanical and Process Engineering, ETH Zurich, Zurich 8092, Switzerland (e-mail: verena.maiwald@micro.mavt.ethz.ch).

This paper has supplementary downloadable material available at http://ieeexplore.ieee.org., provided by the author.

Color versions of one or more of the figures in this paper are available online at http://ieeexplore.iee.org.

Digital Object Identifier 10.1109/JMEMS.2017.2745051 systems with limited accessibility [1]-[3]. This paper builds on the preliminary data presented in [4] on a broadband, in-plane MEMS amplifier for the detection of very low frequency AE.

In addition to broadband detection, distinction of the wave direction and type (longitudinal or transversal) is used for source localization (e.g. [3]), emphasizing the importance of axis selectivity in acoustic emission sensors. The detection of $\mathrm{AE}$ bursts is usually done by thresholding [1], meaning that a signal is recorded if the output of the sensor exceeds a certain amplitude. The amplitude of the threshold is chosen such that as many AE events as possible are recorded while rejecting spurious signals from environmental noise, which is increasingly large at low frequencies. False positives can be avoided by filtering the signal to the desired frequency band. This can be done in the electronic domain, which causes a large data overhead and increased power consumption. As a low power solution, narrow-band sensors can be employed. However, since AE and MS signals are inherently broadband, these sensors are bound to pick up only a fraction of the target signal. Ideally, the AE sensor itself would be sensitive in a defined frequency band that covers the whole spectrum of the AE signal but rejects other frequency bands, thus relaxing the requirements on electronic filtering.

Conventional sensors employed for the detection of low frequency acoustic and micro-seismic vibration signals in the range of a few up to tens of $\mathrm{kHz}$ are either low frequency piezo ceramic AE sensors, which are heavy and expensive, or linear, broadband accelerometers, which are also sensitive to low frequency environmental noise. The advantages of MEMS based AE sensors could lead to a reduction of size and cost which would enable a large scale application in wire-less sensor networks [5]. Bandlimited sensor transfer functions on the other hand could reduce the influence of environmental noise. Extensive research has been conducted on capacitive sensors with sensitive frequencies $>50 \mathrm{kHz}$ ( [6]- [9]), but also on piezoelectric [10] and optical MEMS AE sensors. Further, MEMS AE sensors can provide information on wave direction, while piezo ceramic transducers are usually only sensitive in the out-of-plane direction. This can be achieved by integrating multiple sensors (in-plane and out-of-plane) on one chip (e.g. [8], [9]).

Whereas highly sensitive resonant (underdamped) capacitive MEMS acoustic emission sensors have been demonstrated e.g. in [7], they are tuned to a single resonance line and are therefore restricted to a small frequency range. A broader coverage of the signal spectrum has been achieved by 
using an array of transducers with different frequencies by Ozevin et al. [6]. Alternatively, Sorger et al. [11] and Auerswald et al. [12] obtained an electro-mechanical bandpass characteristic by exploiting the phase shift between two separate oscillators of different resonance frequency. The disadvantage of relying on resonance for increasing the devices sensitivity are long settling times of the transducer which can lead to undesired downtime in-between the detection of AE/MS events.

Other than using low damping, narrow-band resonant devices, sensitivity can be enhanced over a broad frequency range by using displacement amplifying compliant levers such as in [13] where static amplification factors of $40(32 \mathrm{~dB})$ for a sensing bandwidth of $749 \mathrm{~Hz}$ have been demonstrated. These static approaches have a flat frequency response, which means that they are also amplifying low frequency environmental noise. Additionally, their amplification factor is restricted by the torsional stiffness of the lever anchor.

Multi degree of freedom coupled mass networks have been used for example in MEMS gyroscopes [14] or electrostatic power harvesters [15] with the purpose of dynamically amplifying displacement and broadening of the spectrum. The dynamic amplification is based on the fact that kinetic energy transferred from a heavier and stiffer mass-spring oscillator to a lighter and softer one leads to a larger displacement. These kind of systems found in literature are mostly restricted to a small number of masses such as two or three. Acar et al. for example presented a 3 coupled mass gyroscope in [14], where the masses were coupled such as to achieve dynamic amplification of the driving amplitude (amplification factor of 1.7).

In [16] we introduced a mechanical network achieving both mechanical amplification and band-limited detection. There, up to 8 oscillators with decreasing mass and stiffness were coupled in series to achieve a minimum amplification of $20 \mathrm{~dB}$ over a broad frequency range of 1.6-11.5 kHz. These devices were characterized optically.

The mechanism provides zero power amplification for a defined, broad frequency band, which is promising for the remote detection of vibration as used in structural monitoring. This type of large, multi-degree of freedom structure is prone to some limitations with respect to the application in structural monitoring: Low mechanical stability and reproducibility and spurious gimbal modes within the measurement band. Additionally, it needs to be integrated with an electrical read out. These challenges are addressed in this work.

First of all, we present an in-plane design where the sensitive direction $(y)$ is separated from the direction which is mainly sensitive to stress (z).

This improves the resilience of the device towards (inhomogeneous) residual stress gradients. Large compliant structures are typically sensitive to stress leading to warping of the structure in the $\mathrm{z}$ direction. This makes the definition of readout gaps difficult to control and reproduce in out-of-plane devices.

Additionally, axis selectivity has to be provided despite the high number of degrees of freedom inherent to a coupled-mass based amplifier. Eigenmodes in axes other than the sensing axis - for example gimbal modes (rotational modes around the $\mathrm{x}$ - or $\mathrm{y}$-axis) - occurring inside the desired measurement spectrum have to be avoided. To achieve a uniaxial sensor with high amplification, the design was optimized such that parasitic modes are shifted to higher frequencies.

Further, a differential capacitive read out in an out-ofplane configuration can only be achieved by bonding multiple wafers. The presented in-plane design on the other hand can be fabricated in a single mask silicon on insulator (SOI) process. The differential gap closing variable capacitance provides additional selectivity of the in-plane y-axis motion over out-of-plane z-motion.

Finally, damping is a crucial design parameter in acoustic/micro-seismic MEMS sensors as it is determining the sensitivity at resonance, the settling time and also the linearity of the devices. Whereas closed form solutions exist for simple geometries and have been studied extensively, the influence of damping on complex geometries with multiple modes is not straight-forward. Therefore, the influence of pressure on the performance of a coupled-mass based amplifier is studied in this work.

The paper is structured as follows: In part II, the general device concept is introduced and design guidelines are given for shifting undesired degrees of freedom out of the operational bandwidth. Part III contains the description of the methods used for simulation, fabrication and experiments. Subsequently, the final design and the results of the optical as well as the pressure dependent capacitive measurements are presented in section IV and discussed in section V. Finally, an outlook on the further improvement of the devices is given in VI.

\section{DESIGN}

\section{A. Device Concept}

The amplification and bandwidth was achieved by imposing the following design rules on the mass-spring system with $n$ masses (from [16]):

$$
\begin{aligned}
\omega_{0} & =\sqrt{\frac{k_{i}+k_{i+1}}{m_{i}}}=\sqrt{\frac{k_{n}}{m_{n}}} \\
k_{i+1} & =\frac{1}{2} k_{i},
\end{aligned}
$$

where $\omega_{0}$ corresponds to the natural frequency of a single mass-spring oscillator with stiffness $k_{i}+k_{i+1}$ and mass $m_{i}$. Its value is matched by design (eq. (1)) and constitutes the center of the frequency spectrum of the device. The frequency matching in combination with the growth rate of the springs further determines the masses (given any value for $m_{1}$ ). The fact that springs and masses decrease towards the last resonator (eq. (2)) leads to a tsunami like shoaling of the vibration amplitude towards the last mass similar to the amplification in the locust ear [17]. The amplification factor and the amplification bandwidth is increasing with the number of coupled masses $\mathrm{n}$ in the system which has been demonstrated in [16]. This differentiates the amplification mechanism from resonant devices where the system response is increasing with the quality factor of a specific resonance only. The amplifier is insofar similar to micro-mechanical band-pass filters such as [18], as it consists of a chain of 


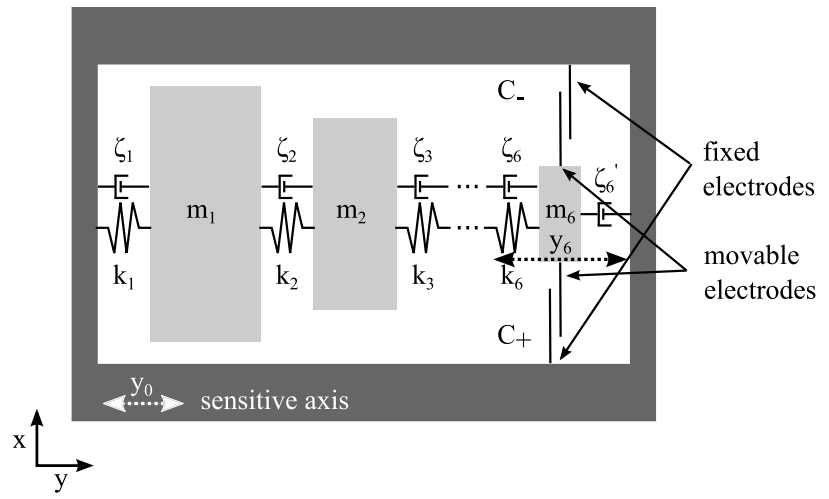

Fig. 1. Lumped element schematic of the transducer. Six masses are coupled in series using design rules (1) and (2), where each mass-spring pair has the same natural frequency and the spring constants are decreasing towards the last mass $\left(m_{6}\right)$. This configuration provides frequency selective mechanical amplification of the package displacement $y_{0}$. The motion of $m_{6}$ (denoted as $y_{6}$ ) can be read out electrically using the attached comb fingers which are arranged to measure the differential capacitive change.

oscillators with identical natural frequency $f_{0}=\omega_{0} /(2 \pi)$. This causes the quasi-bandpass characteristic centered at $f_{0}$ with a pass-band that is depending on the coupling spring between the masses. Instead of merely filtering the incoming mechanical signal by the resonator chain, it amplifies the motion in the pass-band.

The device consists of six masses coupled in series as depicted in fig. 1. The values for masses and springs are derived from the design rules given in equations (1) and (2). When a vibration signal reaches the package $\left(y_{0}\right), m_{1}$ is displaced first, since it is the only mass that couples directly to the package by a spring $\left(k_{1}\right)$. Then, the wave travels further along the intermediate masses $m_{i}$ of the chain until it reaches the last mass $\left(m_{6}\right)$. Due to the decreasing spring constants $k_{i}$ the motion amplitude of each subsequent mass $y_{i}$ is increased. Finally, $m_{6}$ is equipped with a differential capacitance, such that $y_{6}$ can be read out electrically.

\section{B. Design Guidelines}

The design of a single axis-motion sensor requires that offaxis modes are at higher frequencies than the resonances in the sensitive direction (here translational y-axis modes $\left(v_{i}\right)$ ). For typical SOI based beam suspended masses (see fig. 2 (a)) these modes include translational motion in the $\mathrm{x}$-and $\mathrm{z}$-axis $\left(\gamma_{i}\right)$ as well as rotational modes around the $\mathrm{x}-, \mathrm{y}-$ and $\mathrm{z}$-axis $\left(\theta_{i}\right)$. For this design, a number of guidelines were used to shift the z-translational and rotational modes to higher frequencies:

1) Spring Design: Z-translational modes are dominantly influenced by the aspect ratio of the springs $h / t$ where $t$ is the spring width (y) and $h$ the device layer thickness (see fig. 2 (b)). A higher ratio $h / t$ leads to a higher axis selectivity of the device. Since the $y$-axis stiffness of bending beam springs as well as the mass scale linearly with the device layer thickness $h$, the y-axis modes are independent of the device layer thickness. In combination with the fact that the bending stiffness in $\mathrm{z}$-direction $k_{z}$ is proportional to $h^{3}$ for simple beam springs, $\left(\omega_{z} / \omega_{y}\right)^{2}=k_{z} / k_{y}=(h / t)^{2}$ holds, which means that the $S_{y z}$ axis selectivity improves linearly with the thickness of the device layer $h$ given that the beam thickness $t$ remains constant.
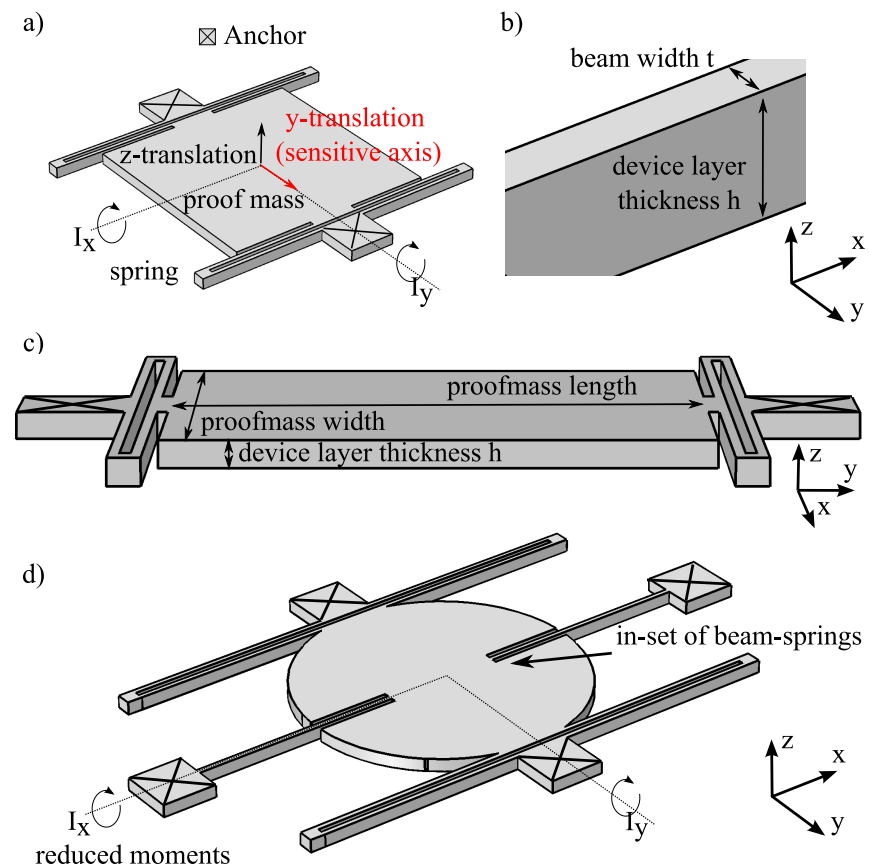

reduced moments

Fig. 2. Illustration of design rules: a) conventional design b) aspect ratio of beam spring c) aspect ratio of proof mass (plate) d) Four instead of two springs provide circumferential clamping of the proof mass. A circular shape reduces the rotational moments $I_{x, y}$. The inset of the beam-springs reduces the device size as well as the effective bending length of the plate-like proof mass.

Given certain fabrication capabilities with respect to the aspect ratio of the structure, spurious modes can be additionally shifted by the lateral design of the structure.

$\mathrm{X}$-axis translational modes as well as rotation around the z-axis occur at high frequencies outside the bandwidth, since they include a longitudinal compression of the beam springs.

The frequency of rotational modes $\theta_{i}$ with frequencies $\omega_{\theta}=\sqrt{\kappa_{i} / I}$ depend of the torsional spring constants $\kappa_{i}$ as well as the moments of inertia of the masses around the respective rotation axis $\left(I_{x}, I_{y}\right)$. Primarily, these modes are shifted by a circumferential placement of springs around the proof mass to increase the torsional stiffness. Further, a large moment of inertia with respect to one of the rotation axes is also undesired. Given a fixed total mass and device layer thickness, the moments of inertia are simultaneously minimized by a circular, concentrically arrangement of the proof mass (fig. 2 (d)). When coupling multiple masses, the in-set of the springs into the masses additionally decreases the devices lateral dimensions and therefore the moments.

2) Plate Stiffness of Proof Mass: In addition to the springs, the compliance of the proof masses themselves is non negligible in large planar devices (see fig. 2 (c)) and their finite plate stiffness $k_{z}^{p, i}$ lowers the frequency of z-translational modes. Considering the proof masses as lumped elements including a lumped stiffness equivalent to their plate stiffness $k_{z}^{p, i}$ connected in series, the total series spring constant is the reciprocal sum of the beam $k_{z}^{b, i}$ as well as the plate stiffness $\left(k_{z}\right)^{-1}=\sum_{i}\left(k_{z}^{b, i}\right)^{-1}+\left(k_{z}^{p, i}\right)^{-1}$. Given a fixed total mass $\mathrm{m}$ and device layer thickness $h$ the stiffness in z-direction $k_{z}^{p, i}$ can be increased by the placement of the spring anchors 
closer to the center of gravity of the device. This reduces the effective bending length of the plate and thereby increases the stiffness (see fig. 2 (d)).

\section{METHODS}

\section{A. Finite Element Analysis}

The design was verified using Finite Element Analysis (FEA). The mode shapes and Eigenvectors were simulated using the built-in eigenfrequency study of COMSOL. The anisotropic elasticity matrix for (100) Si with the (110) planes aligned to the $\mathrm{x}$ and $\mathrm{y}$ axis was used (compare to e.g. [19]). The transfer function presented in section IV, fig. 12 were obtained by frequency domain studies excluding damping effects. The effect of residual stress in the SOI (see Supporting Information) was simulated by a pre-stressed analysis with stress matrix entries $\sigma_{x x}=\sigma_{y y}=\sigma_{0}-\sigma_{1}(z /(h / 2))$ where $\sigma_{0}$ is the mean stress and $\sigma_{1}$ the stress gradient analog to [20]. FEA was also used to simulate the capacitive change of the closing-gap electrodes including fringe fields by solving the electro-statics of the system for a displacement sweep of the last mass $\left(m_{6}\right)$ in $\mathrm{y}$ and $\mathrm{z}$ direction. The resulting capacitances for displacements in $\mathrm{y}$ and $\mathrm{z}$ were also used to extract the capacitive cross-sensitivity $S_{y z}$.

\section{B. Lumped Element Model}

1) Model: In addition to the three-dimensional finite element modal analysis, the motion of the sensor in the sensitive y-axis was studied by a lumped element model. The mechanical transfer function is defined by the solution of the equations of motions of a general multiple degree of freedom system with forced excitation (see e.g. [21]) with the system matrices $[\mathrm{M}]$ (mass matrix), [K] (spring matrix) and $[\mathrm{C}]$ (damping matrix):

$$
\begin{aligned}
{\left[-\omega^{2}[M]+i \omega[C]+[K]\right] \vec{Y} e^{i \omega t} } & =\vec{F} e^{i \omega t} \\
{[A(\omega)] \vec{Y} e^{i \omega t} } & =\vec{F} e^{i \omega t} \\
\vec{Y} & =[A(\omega)]^{-1} \vec{F}
\end{aligned}
$$

For the system presented here the matrices take the form analog to fig. 1: $[M]=\operatorname{diag}\left(m_{1}, \ldots, m_{6}\right)$,

$$
[K]=\left(\begin{array}{cccc}
k_{1}+k_{2} & -k_{2} & & 0 \\
-k_{2} & \ddots & \ddots & \\
& \ddots & k_{5}+k_{6} & -k_{6} \\
0 & & -k_{6} & k_{6}
\end{array}\right)
$$

and

$$
[C]=\left(\begin{array}{cccc}
\zeta_{1}+\zeta_{2} & -\zeta_{2} & & 0 \\
-\zeta_{2} & \ddots & \ddots & \\
& \ddots & \zeta_{5}+\zeta_{6} & -\zeta_{6} \\
0 & & -\zeta_{6} & \zeta_{6}+\zeta_{6}^{\prime}
\end{array}\right)
$$

where $\zeta_{i}$ is the squeeze-film damping between $m_{i}$ and $m_{i-1}$ and $\zeta_{6}^{\prime}$ is the squeeze-film damping between the closing-gap electrode acting on $m_{6}$ only.

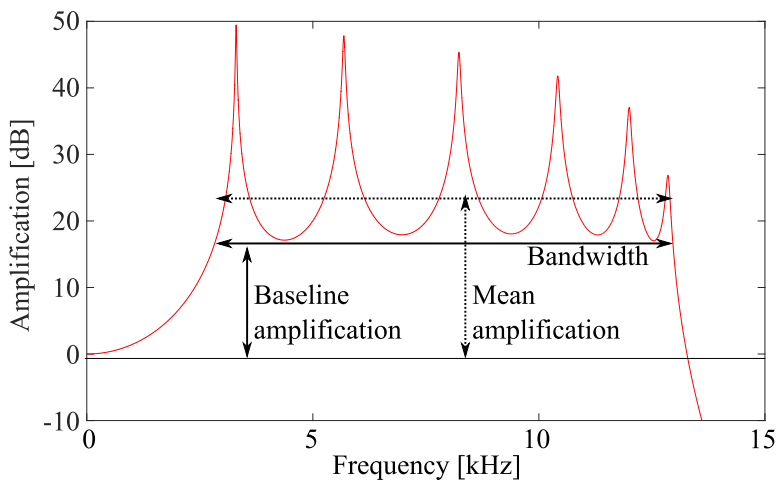

Fig. 3. The mechanical transfer function $y_{6} / y_{0}$ of the lumped element model. Input displacements with frequencies within the device's bandwidth are amplified with a minimum baseline amplification.

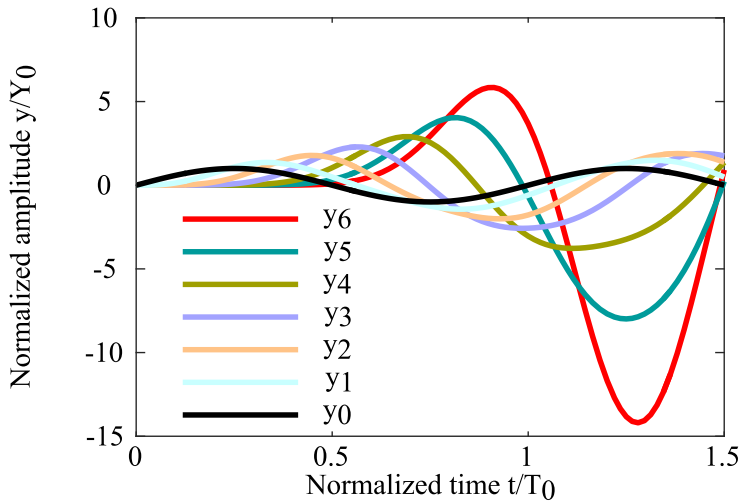

Fig. 4. Lumped element model of the off-resonant, time dependent response to a sine input of $4.65 \mathrm{kHz}$ between the first and second resonance (axes normalized with respect to the input signal amplitude $Y_{0}$ and period $T_{0}$ ).

The squeeze-film damping between the masses is assumed to be proportional to the perimeter of each mass and we define

$$
\zeta_{i}=\zeta \frac{P_{i}}{P_{6}}
$$

where $P_{i}$ is the perimeter of the $\mathrm{i}$-th mass given by the geometry. This assumption reduces the number of free parameters for the damping matrix from 7 to 2 , leaving $\zeta$ as damping coefficient common to all masses and $\zeta_{6}^{\prime}$ as additional damping on $m_{6}$ caused by the squeeze film of the read-out closinggap electrode. Slide-film damping was neglected, since it was estimated from the geometry to be one order of magnitude smaller than the squeeze film damping. Elastic effects are also not included in the model due to the low squeeze number of the geometry.

Fig. 3 shows a transfer function resulting from equation 5 . As device performance parameters the baseline and mean amplification as well as the bandwidth were defined. The baseline amplification is the minimum amplification between the first and last resonance. The bandwidth is the frequency band over which that minimum is achieved, and the mean amplification is the average amplification within said bandwidth. The off-resonant transient motion of the different masses $m_{i}$ is shown in fig. 4. There, the structure is subjected to a sine input of amplitude $Y_{0}$ and period $T_{0}$ with a frequency between the first and second resonance (here $4.65 \mathrm{kHz}$ ). The motional amplitude of the last mass $y_{6}$ is largest. 


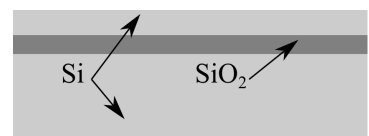

(a)

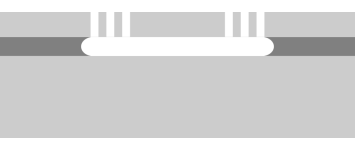

(c)

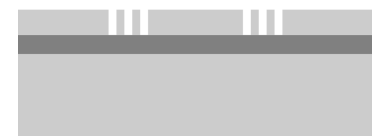

(b)

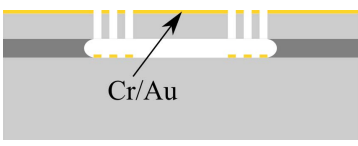

(d)
Fig. 5. a) The masses were fabricated in a one mask SOI based release process. b) ICP dry etch c) $\mathrm{HF}$ vapor release d) evaporation of $\mathrm{Cr} / \mathrm{Au} 4 / 40 \mathrm{~nm}$ for electrical contacts.

As a second stage of the sensor's transfer function, the differential capacitive change in the normalized displacement $\hat{y}_{6}=y_{6} / d_{1}$, with $d_{1}$ being the close gap of the differential gapclosing electrode, was approximated by a Taylor expansion up to 3 rd order

$$
\Delta C=c_{1} \cdot \hat{y}_{6}+c_{3} \cdot \hat{y}_{6}{ }^{3},
$$

where the even terms are neglected due to the differential setup (see also II).

2) Model Parameter Extraction: The model parameters were obtained by combining data from simulation and experiment. The mass matrix $[\mathrm{M}]$ and spring matrix $[\mathrm{K}]$ were obtained by FEA simulation of the individual mass-spring pairs. The damping matrix [C] was obtained by using equation 8 and fitting the two parameters $\zeta$ and $\zeta_{6}^{\prime}$ to the six experimentally found quality factors from the capacitive measurement. The polynomial (9) was fitted to the differential capacitive change for y-motion simulated by FEA.

\section{Fabrication}

The devices were fabricated in a single mask SOI process (see fig. 5). First a $\mathrm{SiO}_{2}$ mask was patterned using RIE dry etching. Then the vertical trenches were etched into the $20 \mu \mathrm{m}$ thick (100) device layer by ICP dry etching. Subsequently, the movable structures were released using vapor HF etching. As adhesion layer for the Au-wire bonds $4 / 40 \mathrm{~nm}$ of $\mathrm{Cr} / \mathrm{Au}$ were evaporated on top of the structure after release. Finally the devices were die- and wire-bonded to a ceramic package.

\section{Characterization Setup}

1) Optical Measurement of Unpackaged Devices: In order to identify the mechanical modes in $y$ - and z-direction, the mechanical transfer function of an unpackaged device (device A) was characterized first optically using the Laser Doppler Vibrometer (LDV, Polytec MSA 400). The device was placed in a controlled pressure chamber and actuated by a Piezoelectric Transducer (PZT, in-plane for y-modes and out-of-plane for z-axis modes) and the substrate motion in $\mathrm{y}$ - and z-axis $\left(y_{0}, z_{0}\right)$ and the motion of $m_{6}\left(y_{6}, z_{6}\right)$ were measured. For the in-plane measurement the stroboscopic Planar Motion Analyzer (PMA) mode was used. The measurements were conducted at a pressure of 3 mbar.

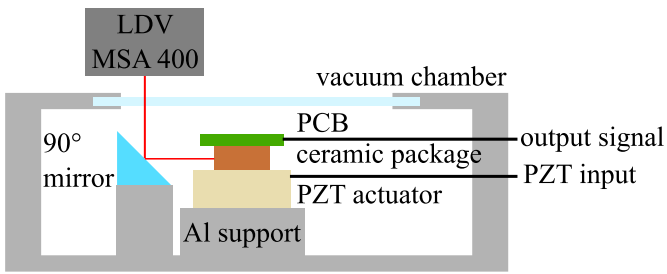

Fig. 6. The measurements were conducted in a controlled pressure chamber. The mechanical motion of the chip package was characterized optically by the Laser Doppler Vibrometer (LDV) simultaneously with the capacitive output signal.
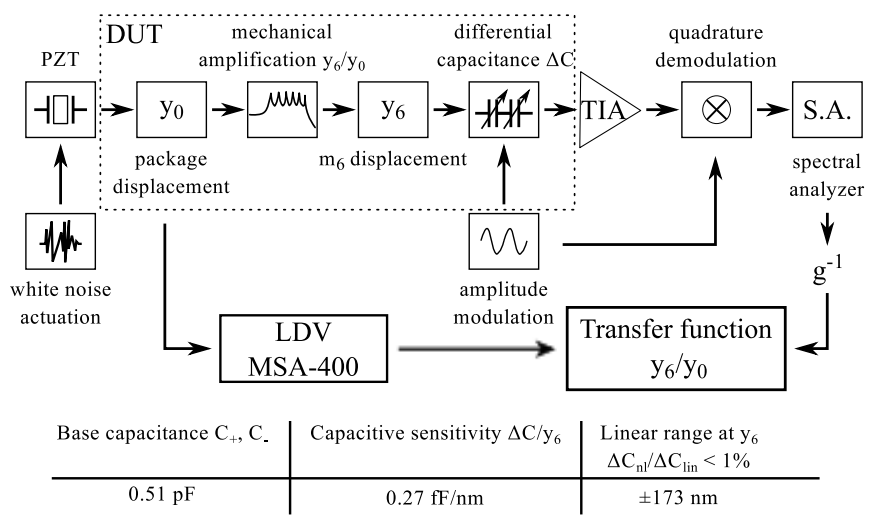

Fig. 7. Signal path of measurement. A shear PZT was actuated by a white noise voltage signal. The resulting motion of the chip package was characterized by the MSA $400 \mathrm{LDV}$ and used as the input signal for the transfer function. At the same time the capacitive output was obtained by an AM circuit with a transimpedance amplifier.

2) Capacitive Characterization of Packaged Devices: The capacitive measurement was conducted on a packaged device of the same batch and design (device B). The ceramic package was flip-mounted on an in-plane PZT actuator and placed inside a controlled pressure chamber (fig. 6). The schematic of the measurement is displayed in fig. 7. The device was actuated mechanically by a white-noise signal on the PZT. To account for acoustic attenuation of the mounting, the spectrum of the package input displacement $y_{0}$ was measured in-situ using the LDV and a right angle mirror. The capacitive spectrum was measured using amplitude modulation at $95 \mathrm{kHz}$ and the built-in quadrature demodulation of a Lock-In amplifier (Zurich Instruments). The gain of the capacitive amplitude modulation circuit was $g=\frac{g_{T I A}}{\sqrt{2}} V_{m} c_{1}\left(\omega_{m}+\omega_{s}\right)$ where $g_{T I A}$ is the gain of the trans-impedance amplifier $V_{m}$ the modulation voltage, $c_{1}$ the linear capacitive change per displacement of $m_{6}$ obtained by FEA simulation, $\omega_{m}$ the modulation frequency and $\omega_{s}$ the signal frequency. Then $y_{6}$ was obtained by $g^{-1} \cdot V_{s}$, where $V_{s}$ is the output of the demodulator.

The quality factors of the capacitive measurements were extracted by fitting a Lorentzian peak to the individual resonances and dividing the peak position by the frequency difference where the peak amplitude decays to $-3 \mathrm{~dB}$.

\section{RESUlTS}

\section{A. Design Freeze}

The realized design is a concentrically arrangement of the proof masses with $m_{1}$ as the outermost mass and $m_{6}$ and the electrodes in the center, following the design rules given in II-B (see fig. 8). The designed aspect ratio of the springs 


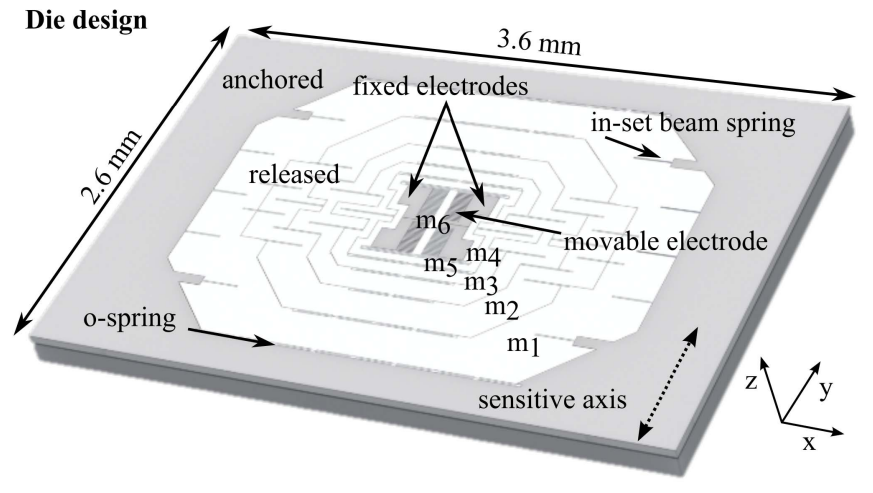

Fig. 8. Die design and top-view of the six proof masses coupled in series. The masses are concentrically arranged with the readout electrodes at the center.

$h / t$ is 6.7. The aspect ratio of the lateral device extent to the device layer thickness is 111 . The form of the masses deviates from the circular shape to accommodate the springs and to avoid sharp angles during fabrication. The simulated mode shapes and resulting lumped element Eigenvectors are shown in fig. 9. As desired, the first out-of-plane mode $\gamma_{1}$ has a higher frequency $(18.3 \mathrm{kHz})$ than the six in-plane modes $v_{i}$. The simulation also shows the amplification of the motion towards the center of the device.

A successfully released and bonded device can be seen in fig. 10 .

\section{B. Optical Measurement of Mode Separation}

The optical measurement presented in fig. 11 shows the position of the first $6 \mathrm{y}$-modes $\left(v_{i}\right)$ in a bandwidth of $3-13 \mathrm{kHz}$ and the first translational out-of-plane mode $\left(\gamma_{1}\right)$ which is at a higher frequency $(16.37 \mathrm{kHz})$. Because of the significantly worse resolution of the PMA with respect to the normal vertical displacement readout (z), the motional input amplitude was increased to resolve the in-plane displacement. At $1.4 \mu \mathrm{m}$ of displacement the masses run into the stoppers that limit the motion leading thus to the trimmed resonance peaks seen in fig. 11. Whereas the first z-mode is separated from the first six in-plane modes as desired, the resonance frequencies are at significantly lower values than obtained by FEA (see section V and Supporting Information).

\section{Capacitive Measurement}

The capacitive transfer function for a measurement conducted at 6.3 mbar is displayed in fig. 12. The bandwidth and peak positions agree with the optical characterization of device $\mathrm{A}$ and the refined FEA simulation. Both ICP-DRIE overetch and stress in the refined FEA are required to obtain the level of agreement between theory and experiment shown in table I. The measured transfer function matches the simulated ones after model refinement with only a $\pm 90 \mathrm{~Hz}$ deviation of the position of the peaks or maximum $2.5 \%$ resonance frequency accuracy (fig. 12, table I). The corrected FEA model was used for extracting the capacitive parameters. The capacitive crosssensitivity $S_{y z}$ over the motion range of the device was also extracted from FEA and calculated to be $0.06 \%$.
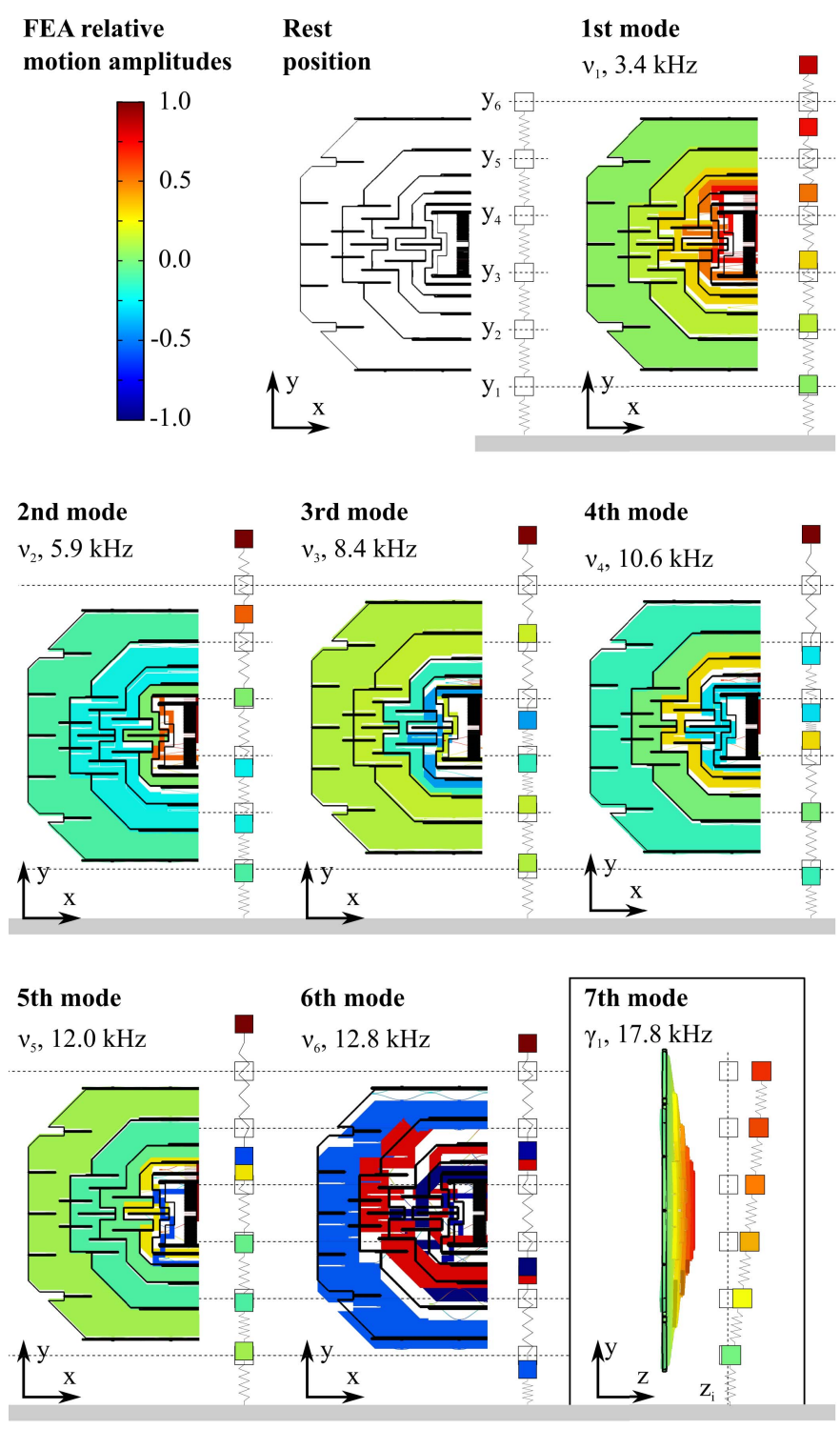

Fig. 9. Representation of the first 7 Eigenmodes of the resonator chain. For each Eigenmode the top view of FEA simulated mode shapes (left side) is shown together with a one dimensional representation of the corresponding Eigenvectors (right side, arbitrary displacement scale), where the dashed lines represent the rest position of the i-th mass. The first six Eigenmodes are in y-direction $\left(v_{i}\right)$ whereas spurious modes (z-axis translational modes $\gamma_{i}$ and rotational modes around the $\mathrm{x}$ - and $\mathrm{y}$-axes $\theta_{i}$ ) are at higher frequencies, the first one being a z-mode $\gamma_{1}$. The center mass $\left(m_{6}\right)$ provides the highest displacement wrt. the rest position.

At frequencies higher than the mechanical roll-off the signal is not dropping completely which can be explained by the cubic nonlinearity of the read out capacitance (see Supporting Information). From the measured transfer function the minimum and mean amplification as well as the bandwidth of the amplifier were extracted. The minimum amplification $g_{\min }=16.4 \mathrm{~dB}$ is defined as the minimum output between $v_{1}$ $(3.32 \mathrm{kHz})$ and $v_{6}(12.79 \mathrm{kHz})$, the amplification bandwidth is the frequency range over which this minimum amplification is achieved $(10 \mathrm{kHz}$ over a range from $3-13 \mathrm{kHz})$ and the mean amplification $g_{\text {mean }}=23.5 \mathrm{~dB}$ the mean over this bandwidth. The characteristic frequency $f_{0}=7.94 \mathrm{kHz}$ is defined as the center of the bandwidth. 
TABLE I

Comparison FEA Modes With Capacitive Measurement

\begin{tabular}{|c||c||c||c|c|}
\hline \multicolumn{1}{|c||}{ Mode No. } & \multicolumn{3}{|c|}{$\begin{array}{c}\text { FEA } \\
{[\mathrm{kHz}]}\end{array}$} & $\begin{array}{c}\text { Measurement } \\
{[\mathrm{kHz}]}\end{array}$ \\
\hline \hline y-mode & $\begin{array}{c}\text { as } \\
\text { designed }\end{array}$ & $\begin{array}{c}\text { ICP-DRIE } \\
\text { overetch }^{1}\end{array}$ & $\begin{array}{c}\text { ICP-DRIE } \\
\text { overetch } \\
\text { and stress }\end{array}$ & capacitive \\
\hline$f_{0}$ & 11.50 & 7.06 & 8.12 & 7.94 \\
\hline$\nu_{1}$ & 4.67 & 2.84 & 3.40 & 3.32 \\
\hline$\nu_{2}$ & 8.08 & 4.95 & 5.86 & 5.77 \\
\hline$\nu_{3}$ & 11.69 & 7.16 & 8.40 & 8.32 \\
\hline$\nu_{4}$ & 14.85 & 9.07 & 10.55 & 10.52 \\
\hline$\nu_{5}$ & 17.24 & 10.47 & 11.99 & 12.00 \\
\hline$\nu_{6}$ & 18.53 & 11.47 & 12.81 & 12.79 \\
\hline \hline z-mode & $\begin{array}{c}\text { as } \\
\text { designed }\end{array}$ & $\begin{array}{c}\text { ICP-DRIE } \\
\text { overetch }\end{array}$ & $\begin{array}{c}\text { ICP-DRIE } \\
\text { overetch } \\
\text { and stress }\end{array}$ & \\
\hline$\gamma_{1}$ & 19.39 & 17.18 & 17.78 & LDV \\
\hline
\end{tabular}

${ }^{1}$ including $-450 \mathrm{~nm}$ overetch from designed layout

${ }^{2}$ Mean stress $\sigma_{0}=12.7 \mathrm{MPa}$, stress gradient $\sigma_{1}=0.92 \mathrm{MPa}$

Both overetch as well as static strain were obtained from optical measurements (SEM and White Light Interferometry, see Supporting Information).
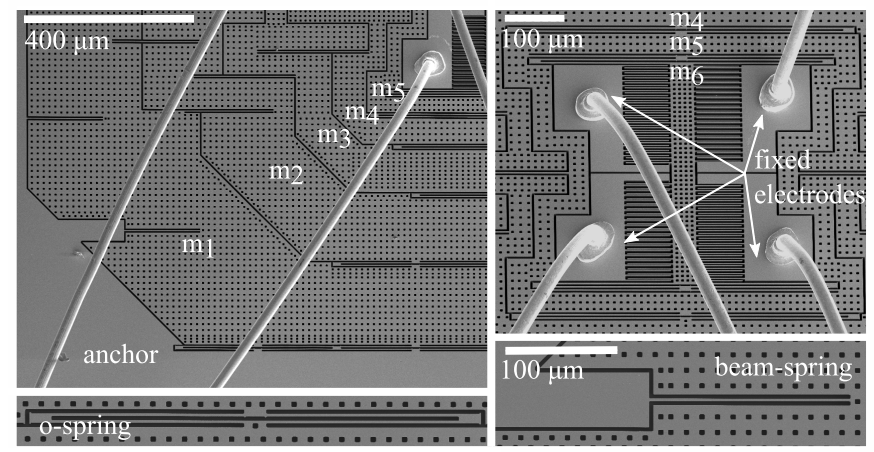

Fig. 10. SEM image of the fabricated device. Au-wire bonds provide electrical connection to the fixed electrodes.

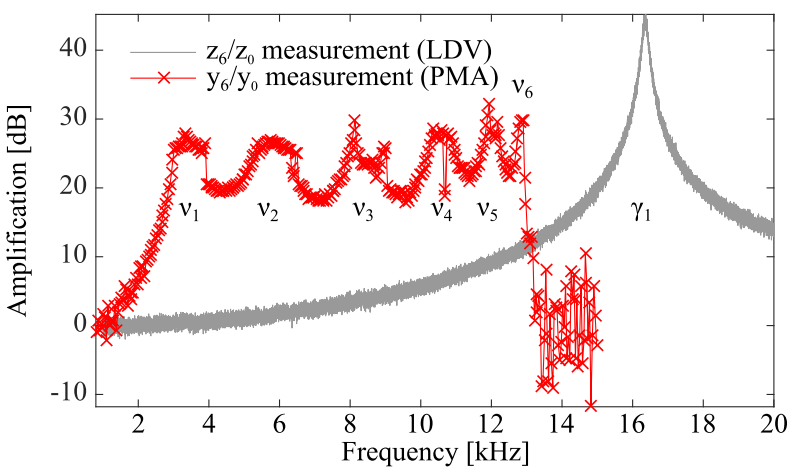

Fig. 11. Optical measurements of transfer function of $y_{6} / y_{0}$ and $z_{6} / z_{0}$ of device A. The out-of-plane measurement shows that the first z-peak $\gamma_{1}$ is located outside the target frequency range $\left(3-13 \mathrm{kHz}\right.$ for $v_{1}-v_{6}$, minimum amplification $18 \mathrm{~dB}$ ). In-plane measurements were performed using the Planar Motion Analyzer stroboscopic measurement technique. The measured resonances are shifted to lower frequencies compared to the FEA model.

\section{Damping and Pressure Dependence}

The operational range was identified by measuring at different pressure set-points between 6.3 mbar and ambient pressure. The peak amplitudes decrease significantly with increasing

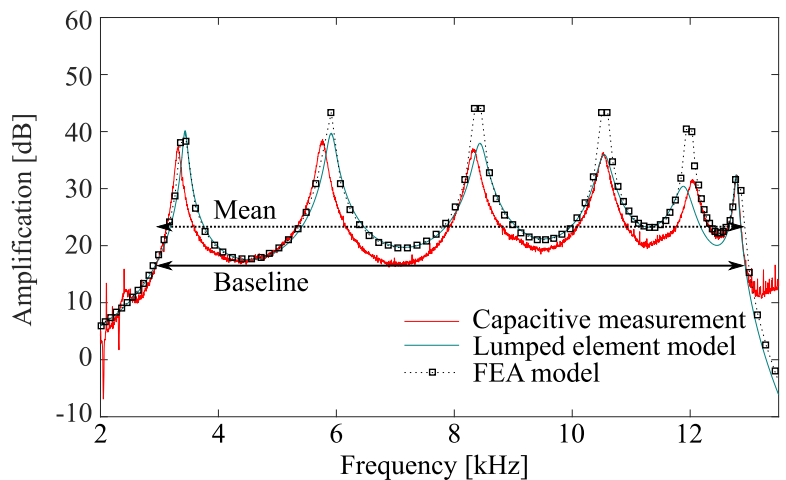

Fig. 12. Capacitive measurement vs. model. The position and amplitude of the peaks are in good agreement with the model $( \pm 90 \mathrm{~Hz}$, see also table (I)). The amplification provided in the frequency band of $3 \mathrm{kHz}-13 \mathrm{kHz}$ is a minimum of $16.4 \mathrm{~dB}$ and a mean of $23.5 \mathrm{~dB}$. The capacitive measurement was conducted at a pressure of 6.3 mbar. The deviation at high frequencies can be explained by the cubic non-linearity of the read-out capacitance.

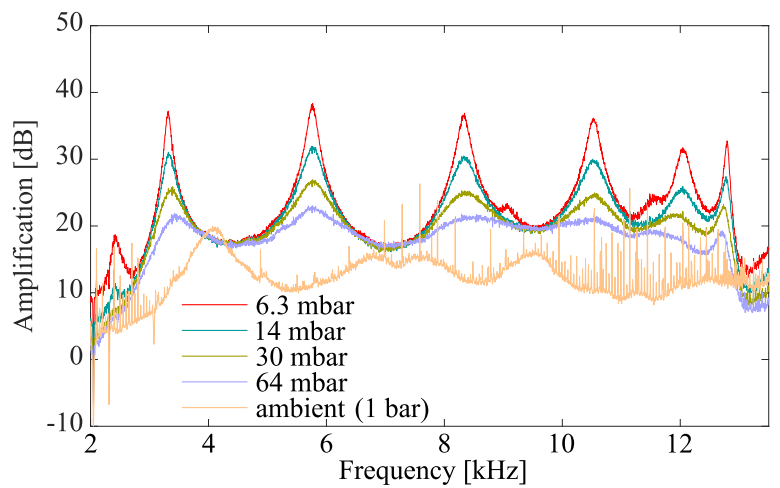

Fig. 13. The pressure dependence of the transfer function. A moderate increase in pressure leads to a reduced quality factor of the modes, while the minimum amplification is preserved. A further increase of the damping leads to a reduction of the amplitude at higher modes. At ambient pressure the response of the device is strongly suppressed.

pressure (see fig. 13) while the baseline is only reduced by less than $10 \%$ up to a pressure of 64 mbar. The baseline starts to deteriorate first at high pressures between the 5th and the 6th mode. At ambient pressure the motion of $m_{6}$ is strongly suppressed due to the squeeze film between the comb fingers of the last mass and the readout. Minimum and mean amplification as well as bandwidth have been extracted for all pressure values and are displayed in fig. 14 .

\section{E. Lumped Element Model}

The two damping coefficients of the lumped model, the perimeter proportional intra-mass damping $\zeta$ and the additional damping from the closing-gap electrode $\zeta_{6}^{\prime}$ were fitted to the experimental quality factors (given in fig. 15) to obtain the damping matrix $[\mathrm{C}]$ (see equation 7 ). The resulting transfer function based on the parameters in table II reproduces well the experiment as can be seen in fig. 12 .

\section{DisCusSION}

In this section we review the performance of the device with respect to the application. This includes the filtering and amplification properties as well as the influence of damping on the device parameters. An overview of the device specifications is given in table III. Further, we evaluate the influence of 


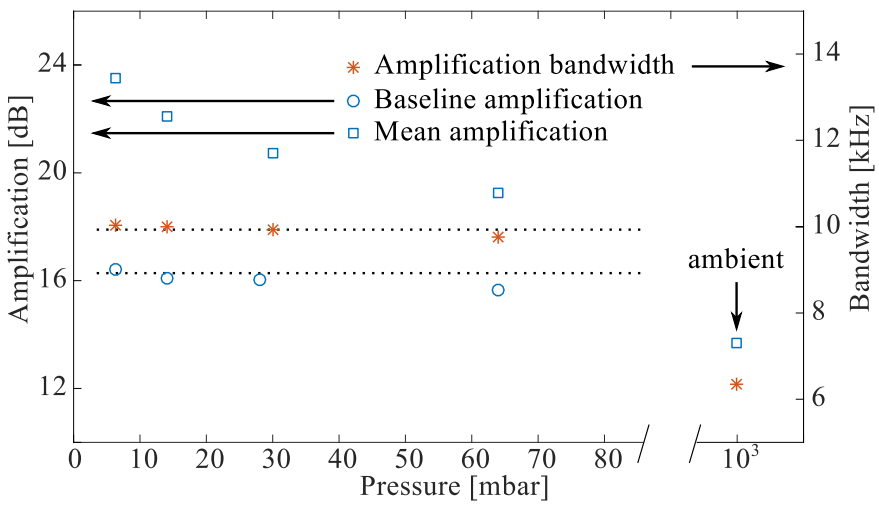

Fig. 14. Baseline amplification and bandwidth of the device showed a decrease of less than $10 \%$ for a wide pressure range between 6.3 and 64 mbar. The mean amplification is sensitive to the peak amplitudes and therefore to an increase in damping. At ambient pressure the device performance decreases.

TABLE II

LUMPED ELEMENT MODEL

\begin{tabular}{|c|c|}
\hline System parameter & Value \\
\hline Spring vector $\vec{k}[\mathrm{~N} / \mathrm{m}]$ & {$[194,78,36,16,8,3]$} \\
\hline Mass vector $\vec{m}[\mu \mathrm{g}]$ & {$[62.0,31.0,15.5,7.7,3.6,1.0]$} \\
\hline Intra-mass damping $\zeta \frac{P_{i}}{P_{6}}\left[\mathrm{Nsm}^{-1}\right]$ & $0.38 \mathrm{e}-6 \cdot[9.0,8.1,5.6,4.9,3.0,1]$ \\
\hline $\begin{array}{c}\text { Damping closing-gap electrode } \\
\zeta_{6}{ }^{[}\left[\mathrm{Nsm}^{-1}\right]\end{array}$ & $4.7 \mathrm{e}-6$ \\
\hline Capacitive fit coefficients $\left[c_{1}, c_{3}\right][\mathrm{F}]$ & {$[5.12 \mathrm{e}-013,6.42 \mathrm{e}-013]$} \\
\hline
\end{tabular}

TABLE III

DEVICE SPECIFICATIONS

\begin{tabular}{|c|c|}
\hline Die size & $3600 \mu \mathrm{m} \times 2600 \mu \mathrm{m}$ \\
\hline Device extent & $1642 \mu \mathrm{m} \times 2222 \mu \mathrm{m}$ \\
\hline Device layer & $19.6 \mu \mathrm{m}$ \\
\hline$m_{1}$ & $62 \mu \mathrm{g}$ \\
\hline$m_{6}$ & $1.0 \mu \mathrm{g}$ \\
\hline$d_{1}$ & $1.9 \mu \mathrm{m}$ \\
\hline$d_{2}$ & $4.9 \mu \mathrm{m}$ \\
\hline$C_{+}, C_{-}$ & $0.51 \mathrm{pF}$ \\
\hline$\Delta C$ at $y_{6}$ & $0.06 \%$ \\
\hline Capacitive cross sensitivity $S_{y z}$ & $0.27 \mathrm{fF} / \mathrm{nm}$ \\
\hline Linear range at $y_{6}\left(\frac{\Delta C_{n l}}{\Delta C_{l i n}}<1 \%\right)$ & $\pm 173 \mathrm{~nm}$ \\
\hline Center frequency $f_{0}$ & $7.94 \mathrm{kHz}$ \\
\hline Amplification bandwidth & $10 \mathrm{kHz}$, range $3 \mathrm{kHz}-13 \mathrm{kHz}$ \\
\hline Baseline amplification & $16.4 \mathrm{~dB}$ \\
\hline Mean amplification at $6.3 \mathrm{mbar}$ & $23.5 \mathrm{~dB}$ \\
\hline
\end{tabular}

damping and process variations by means of FEA and lumped element modeling.

\section{A. Performance as Mechanical Filter}

The transfer-function presented in section IV can be evaluated with respect to band-pass filter characteristics. Within the operational bandwidth the sensitivity of the amplifier is a minimum of $16.4 \mathrm{~dB}(6.6 \mathrm{~nm} / \mathrm{nm})$ whereas at lower frequencies the mechanical gain is $0 \mathrm{~dB}$. This corresponds to a relative rejection of $16.4 \mathrm{~dB}$. Rejection of lower frequency bands is important, since this is where environmental noise is

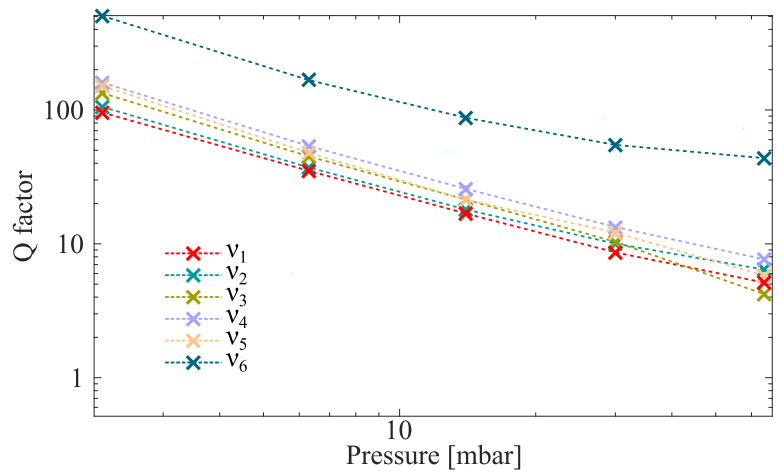

Fig. 15. Quality factors of in-plane peaks $v_{i}$ for the capacitive measurement. The values were derived by dividing the peak position by the frequency difference where the resonance peak amplitude decays to $-3 \mathrm{~dB}$. The error in the Q-factors due to uncertainties in peak positions and widths is in the order of 1 .

the strongest. Additionally peak-to-peak ripple can be used as a measure for the flatness of the filter response within the operational bandwidth. In this case the minimum minus maximum amplitude within the operational bandwidth, for bandpass filters usually called peak-to-peak ripple, is depending not only on the spacing of the resonances given by the design but also on the pressure in the measurement chamber. Our measurements show that it can be reduced down to $11.9 \mathrm{~dB}$ by increasing the pressure to 64 mbar while sacrificing only $10 \%$ of the baseline amplification. At lower pressures the pk-pk ripple is much higher (e.g. $21.95 \mathrm{~dB}$ at $6.3 \mathrm{mbar}$ ). The amplified motion on the last mass leads to an enhanced relative capacitive signal $\Delta C / C_{0}$ wrt. the base capacitance $C_{0}$. In contrast, configurations of mechanically un-coupled sensor-arrays that are connected in parallel in the electrical domain (similar to [12]) achieve an increased signal by a higher base capacitance $C_{0}$ of the system (see also Supporting Information). This distinction may prove beneficial for certain system aspects, such as read out circuits and energy efficiency.

\section{B. Response Time and Quality Factors}

The response time and ring down time of a AE/MS sensor is important for co-detection and the dead-time of the sensor after the detection of an event. The initial mechanical response time depends on the time the signal takes to travel from the package to the center-mass (see fig. 4). After that, the damping determines how long the system takes to reach steady state. In principle higher damping is favorable for avoiding long ring down times. One measure for the ring-down time is the $\mathrm{Q}$ factor of the resonance modes, which are displayed in fig. 15. The error in determining the $Q$ factors of the individual increases with pressure due to the superposition of multiple peaks with low quality factors. The quality factor of the last mode $v_{6}$ is distinctively higher than the others, which we attribute to its modeshape (compare to fig. 9), that involves comparably large motional amplitudes of the outer masses and thereby a higher stored energy in the system. The outer masses are subject to lower damping $\left(\zeta_{i}\right)$ than the center mass $m_{6}\left(\zeta_{6}+\zeta_{6}^{\prime}\right)$ which in turn leads to a higher quality factor. The values extracted for this mass-spring network are in the 
order of 100 for a pressure of 6.3 mbar and below 10 (with the exception of the last mode) for a pressure of 64 mbar, yielding a reasonable settling time of the system. The values for high pressures are comparable to the $\mathrm{Q}$ factors of 5-15 presented in Ozevin et al. [6].

\section{Damping Model}

As mentioned above, damping is an important parameter in the performance of MS/AE sensors. Therefore, a model to approximate the influence of pressure on the damping characteristic is required, even for a system with a high number of degrees of freedom such as the one presented in this work. The standard approach for the computation of damping parameters as used in e.g. [7] is to use closed form expressions for simple plate structures or perforated plates or FEA simulation. The underlying assumptions for the analytic models, e.g. a perpendicular motion are oversimplifying in a multi-degree of freedom structure such as this one with many masses, shear motion and multiple different modes and damping mechanisms (such as slide-film and squeeze film damping). Also, FEA damping simulation require very high computational power. This makes it difficult to relate the experimentally observed quality factors with the pressure set-points.

The approach followed here to retrieve the damping coefficients relies on the assumption of the form of the damping matrix [C] (see equation 7) and a reduction of free parameters to two dominant ones.

The two fitted damping coefficients for the squeeze-film damping between the comb-fingers $\zeta_{6}^{\prime}$ and between the masses $\zeta$ can be compared to analytical solutions of a parallel plate squeeze film model. The Knudsen numbers $K_{n}$ for the geometry vary from 0.3 to 16 for the measured pressures (except ambient) which are commonly attributed to the transition regime [22], where rarefied gas models are applicable. Assuming an effective viscosity model and a function of $K_{n}$ as found by [23] $\mu_{\text {eff }}=\frac{\mu}{1+9.638 K_{n}^{1.159}}$ together with the solution to the Reynolds equation for an incompressible rectangular squeezefilm presented in [24], the damping coefficients were computed to be $\zeta=0.02 \cdot 10^{-6} \mathrm{Ns} / \mathrm{m}$ and $\zeta_{6}^{\prime}=0.5 \cdot 10^{-6} \mathrm{Ns} / \mathrm{m}$ for a pressure of 6.3 mbar. Despite the fact that these closed form models are a strong simplification, they are comparable in magnitude to the fitted values of the 6 DOF model given in table II. Also the shape of the transfer-function of the lumped element model matches well the experimental one.

\section{Axis Selectivity}

Design rules were developed for obtaining axis-selectivity for a planar sensor with a high number of degrees of freedom. A compact concentrically arranged design with minimized moments was used to suppress rotational modes. The outof-plane translational modes are proportional to the aspect ratio of the beam springs which was maximized within the ICP dry etching capabilities. In addition, the finite stiffness of the masses themselves was increased by the placement of the spring-anchors close to each other. As a result, the first outof-plane mode was located outside the operational bandwidth.
In addition the gap-closing differential capacitive readout provides axis selectivity in the electrical transduction with a low cross-sensitivity $S_{y z}$ of $0.06 \%$. In combination this provides a good selectivity of the sensitive in-plane axis over out-of-plane motion.

\section{E. Influence on Process Variation on Device Performance}

Process variations such as mask undercut during the ICP dry etch and residual stress in the structure can influence the performance of the device by causing a frequency mismatch of the individual resonators, thus violating equation (1). Whereas over-etching reduces the stiffness and mass of the device, stress increases the stiffness (see table I), counteracting each other. Both effects where included in the FEA and lumped element model (details can be found in the Supporting Information)

\section{CONCLUSION}

This work demonstrates an in-plane broadband (3-13 kHz) vibration amplifier with improved transfer function and differential capacitive read-out. Previous work on coupled mass amplification [16] demonstrated how this mechanism can provide quasi band-pass amplification of motion at zero power expense. These attributes are very attractive for low power detection of acoustic emission signals in high attenuation materials e.g. for structural health monitoring (rock, bridges, pipelines, cliffs etc.). The amplification mechanism relies on a chain of frequency matched oscillators with decreasing springs and masses which results in an off-resonant amplification within the operational bandwidth that is not dependent on the quality factor of the mechanical resonances.

The use in traditional, higher frequency AE application is also possible by designing for a higher center frequency $\omega_{0}$ in accordance with the presented design rules.

Here, we present an in-plane coupled mass-spring network that despite the high number of degrees of freedom has a high axis selectivity. Optical measurements demonstrate that the spurious z-modes were successfully shifted outside the device's operational bandwidth. The gap-closing differential capacitive readout provides additional selectivity with a crosssensitivity of only $0.06 \%$.

Our measurements show that depending on the pressure in the setup or package, the transfer function is flattened with a minimum amplification of $16 \mathrm{~dB}$ almost independently of pressure. The transfer characteristic at different pressure setpoints between 6.3 mbar and ambient pressure was measured. The FEA and a lumped element model using a damping matrix based on a perimeter proportional squeeze-film damping was compared to the measurement and found to be in good agreement. The peak amplitudes decrease significantly with increasing pressure while the baseline is only reduced by less than $10 \%$ up to a pressure of 64 mbar. Also, the low sensitivity of the minimum amplification towards pressure changes relaxes the constraints of achieving and maintaining vacuum in the package. Therefore, the device is preferably operated in this pressure regime around 30 mbar.

Future work will include the increase of the amplification factor by adding more masses. Maintaining the stability and 
axis selectivity of such an up-scaled design can be achieved on the one hand by increasing the device layer thickness and the aspect ratio of the ICP process and on the other hand by including the bulk of the SOI for defining the outer masses. Additionally, a suitable package for mounting the sensor on the surface under test has to be developed with the possibility to integrate the chip in a 3 -axis configuration.

\section{ACKNOWLEDGMENT}

The authors would like to thank Prof. Dr. Lothar Thiele and Dr. Jan Beutel for fruitful discussions and the ETH FIRST-CLA and BRNC clean room staff (especially Donat Scheiwiller and Ute Drechsler) as well as Martin Lanz from the Department of Electrical Engineering at ETH Zurich for their continuous support with fabrication.

\section{REFERENCES}

[1] G. Michlmayr, D. Cohen, and D. Or, "Sources and characteristics of acoustic emissions from mechanically stressed geologic granular media-A review," Earth-Sci. Rev., vol. 112, nos. 3-4, pp. 97-114, 2012.

[2] G. Senfaute, A. Duperret, and J. A. Lawrence, "Micro-seismic precursory cracks prior to rock-fall on coastal chalk cliffs: A case study at Mesnil-Val, Normandie, NW France," Natural Hazards Earth Syst. Sci., vol. 9, no. 5, pp. 1625-1641, 2009.

[3] X. Cui, Y. Yan, Y. Ma, L. Ma, and X. Han, "Localization of $\mathrm{CO}_{2}$ leakage from transportation pipelines through low frequency acoustic emission detection," Sens. Actuators A, Phys., vol. 237, pp. 107-118, Jan. 2016.

[4] V. Maiwald, M. Müller, C. Ritz, C. Roman, and C. Hierold, "Shoaling vibration amplifier with flattened transfer function and suppressed spurious modes," in Proc. IEEE Int. Conf. Micro Electro Mech. Syst. (MEMS), Las Vegas, NV, USA, Jan. 2017, pp. 1154-1157.

[5] H. Ceylan, K. Gopalakrishnan, S. Kim, P. C. Taylor, M. Prokudin, and A. F. Buss, "Highway infrastructure health monitoring using microelectromechanical sensors and systems (MEMS)," J. Civil Eng. Manage., vol. 19, no. 1, pp. S188-S201, 2013.

[6] D. Ozevin, D. W. Greve, I. J. Oppenheim, and S. P. Pessiki, "Resonant capacitive MEMS acoustic emission transducers," Smart Mater. Struct., vol. 15, no. 6, pp. 1863-1871, Dec. 2006.

[7] H. Saboonchi and D. Ozevin, "MEMS acoustic emission transducers designed with high aspect ratio geometry," Smart Mater. Struct., vol. 22, no. 9, p. 095006, 2013.

[8] H. Saboonchi, D. Ozevin, and M. Kabir, "MEMS sensor fusion: Acoustic emission and strain," Sens. Actuators A, Phys., vol. 247, pp. 566-578, Aug. 2016.

[9] A. W. Harris, I. J. Oppenheim, and D. W. Greve, "MEMS-based highfrequency vibration sensors," Smart Mater. Struct., vol. 20, no. 7, p. 075018, 2011.

[10] G.-H. Feng and W.-M. Chen, "Micromachined lead zirconium titanate thin-film-cantilever-based acoustic emission sensor with poly(N-isopropylacrylamide) actuator for increasing contact pressure," Smart Mater. Struct., vol. 25, no. 5, p. 055046, 2016.

[11] A. Sorger, C. Auerswald, A. Shaporin, M. Freitag, M. Dienel, and J. Mehner, "Design, modeling, fabrication and characterization of a MEMS acceleration sensor for acoustic emission testing," in Proc. Solid-State Sens., Actuators, Microsyst. Conf. (TRANSDUCERS), Barcelona, Spain, Jun. 2013, pp. 726-729.

[12] C. Auerswald, A. Sorger, M. Dienel, A. Shaporin, and J. Mehner, "MEMS acoustic emission sensor with mechanical noise rejection," in Proc. Int. Multi-Conf. Syst., Signals Devices (SSD), Chemnitz, Germany, Mar. 2012, pp. 1-6.

[13] I. Zeimpekis, I. Sari, and M. Kraft, "Characterization of a mechanical motion amplifier applied to a MEMS accelerometer," J. Microelectromech. Syst., vol. 21, no. 5, pp. 1032-1042, Oct. 2012.

[14] C. Acar and A. M. Shkel, "Structural design and experimental characterization of torsional micromachined gyroscopes with non-resonant drive mode," J. Micromech. Microeng., vol. 14, no. 1, pp. 15-25, 2003.

[15] Z. Wong, J. Yan, K. Soga, and A. Seshia, "A multi-degree-offreedom electrostatic MEMS power harvester," in Proc. PowerMEMS, Washington, DC, USA, Dec. 2009, pp. 300-303.
[16] M. Müller, V. Maiwald, M. Kach, C. Hierold, and C. Roman, "A passive micromechanical broadband amplifier for acoustic emission sensing," in Proc. Solid-State Sens., Actuators, Microsyst. Conf. (TRANSDUCERS), Anchorage, AK, USA, Jun. 2015, pp. 1129-1132.

[17] R. Malkin, T. R. McDonagh, N. Mhatre, T. S. Scott, and D. Robert, "Energy localization and frequency analysis in the locust ear," J. Roy. Soc. Interface, vol. 11, no. 90, p. 20130857, 2013.

[18] F. Bannon, J. Clark, and C. T.-C. Nguyen, "High frequency microelectromechanical IF filters," in IEDM Tech. Dig., San Francisco, CA, USA, Dec. 1996, pp. 773-776.

[19] M. A. Hopcroft, W. D. Nix, and T. W. Kenny, "What is the Young's modulus of silicon?" J. Microelectromech. Syst., vol. 19, no. 2, pp. 229-238, Apr. 2010.

[20] W. Fang and J. A. Wickert, "Determining mean and gradient residual stresses in thin films using micromachined cantilevers," J. Micromech. Microeng., vol. 6, no. 3, pp. 301-309, 1996.

[21] R. N. Jazar, "Multi degree of freedom systems, frequency response," in Advanced Vibrations: A Modern Approach, 1st ed. New York, NY, USA: Springer, 2013, p. 235.

[22] S. Hutcherson and W. Ye, "On the squeeze-film damping of microresonators in the free-molecule regime," J. Micromech. Microeng., vol. 14, no. 12, pp. 1726-1733, 2004.

[23] T. Veijola, H. Kuisma, J. Lahdenperä, and T. Ryhänen, "Equivalentcircuit model of the squeezed gas film in a silicon accelerometer," Sens. Actuators A, Phys., vol. 48, no. 3, pp. 239-248, 1995.

[24] M. Bao and H. Yang, "Squeeze film air damping in MEMS," Sens. Actuators A, Phys., vol. 136, no. 1, pp. 3-27, 2007.

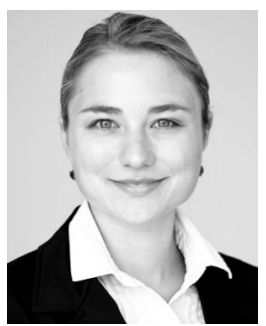

Verena Maiwald received the B.Sc. and M.Sc (Hons.) degrees in physics from the Swiss Federal Institute of Technology, Zurich (ETHZ). During her studies in Zurich and Trinity College Dublin in Ireland, her main interest and focus was experimental solid-state physics. She is currently pursuing the Ph.D. degree with the Group of Micro and Nanosystems, ETH Zurich, with a focus on low power acoustic emission sensing.

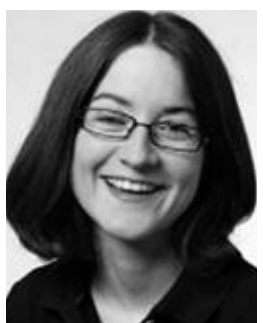

Michelle Müller received the bachelor's and master's degrees in mechanical engineering from the Swiss Federal Institute of Technology (ETHZ), Zurich, Switzerland, where she is focusing on microand nanosystems. In between, she worked eight months with Synthes on implants for the spine. She is currently pursuing the Ph.D. degree with the Micro and Nanosystems Group, ETHZ, with a focus on MEMS acoustic emission sensors.

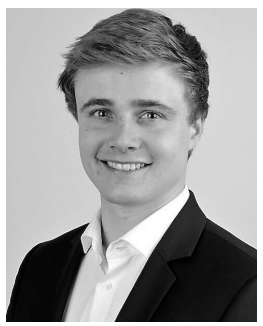

Christian Ritz received the bachelor's degree in mechanical engineering from the Swiss Federal Institute of Technology (ETHZ), Zurich, in 2015. He is currently pursuing the master's degree in applied nanophysics and nanosystems. He wrote his bachelor's thesis with the Micro and Nanosystems Group, ETHZ, where he is currently an Associate Scientist. For one year, he has been part of the Research and Development Team Optotune Switzerland, where he is also an Associate Engineer. 


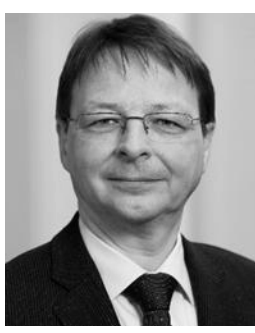

Christofer Hierold (F'17) received the Dr-Ing. degree in engineering Sciences from the Technical University of Munich, Germany, in 1990. He has been a Professor of micro and nanosystems with ETH Zurich, Switzerland, since 2002. He has authored over 230 papers in journals and refereed conference proceedings. He has authored or co-authored over 20 granted patents. He served as the General Co-Chair of MEMS 2009 and the Program Chair of TRANSDUCERS 2013. Since 2012, he has been an Editor-in-Chief of the IEEE

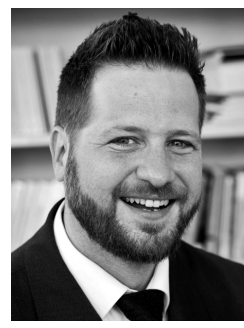
JoURNAL OF MiCROELECTROMECHANICAL SYSTEMS. He is a member of the Swiss Academy of Engineering Sciences.
Cosmin Roman received the M.Sc. degree in electrical engineering from the Polytechnic University of Bucharest, in 2002, and the Ph.D. degree in micro and nanoelectronics from the National Polytechnic Institute of Grenoble, in 2006, where he was involved in modeling carbon nanotube sensors. Since then, he has been a Senior Researcher with ETH Zurich. His current interests include micro and nanotransducers for energy-efficient sensor systems. 